\title{
Network Constraints on Longitudinal Grey Matter Changes in First Episode Psychosis
}

\author{
Sidhant Chopra ${ }^{\mathrm{a}, \mathrm{b}, *}$, Stuart Oldham ${ }^{\mathrm{a}, \mathrm{b}}$, Ashlea Segal ${ }^{\mathrm{a}, \mathrm{b}}$, Alexander Holmes ${ }^{\mathrm{a}, \mathrm{b}}$, \\ Kristina Sabaroedin ${ }^{\mathrm{a}, \mathrm{b}, \mathrm{h}}$, Edwina R. Orchard ${ }^{\mathrm{a}, \mathrm{b}}$, Shona M. Francey ${ }^{\mathrm{c}, \mathrm{d}}$, Brian \\ O'Donoghue $^{\mathrm{c}, \mathrm{d}}$, Vanessa Cropley ${ }^{\mathrm{e}}$, Barnaby Nelson ${ }^{\mathrm{c}, \mathrm{d}}$, Jessica Graham ${ }^{\mathrm{c}, \mathrm{d}}$, Lara \\ Baldwin $^{\mathrm{c}, \mathrm{d}}$, Jeggan Tiego ${ }^{\mathrm{a}, \mathrm{b}}$, Hok Pan Yuen ${ }^{\mathrm{c}, \mathrm{d}}$, Kelly Allott ${ }^{\mathrm{c}, \mathrm{d}}$, Mario \\ Alvarez-Jimenez $^{\mathrm{c}, \mathrm{d}, \mathrm{f}}$, Susy Harrigan ${ }^{\mathrm{c}, \mathrm{d}}$, Ben D. Fulcher ${ }^{\mathrm{i}, \mathrm{j}}$, Kevin Aquino ${ }^{\mathrm{a}, \mathrm{i}, \mathrm{j}}$, \\ Christos Pantelis $^{\mathrm{e}}$, Stephen J Wood ${ }^{\mathrm{c}, \mathrm{d}, \mathrm{g}}$, Mark Bellgrove ${ }^{\mathrm{a}}$, Patrick McGorry ${ }^{\mathrm{c}, \mathrm{d}}$, \\ Alex Fornito ${ }^{\mathrm{a}, \mathrm{b}}$
${ }^{a}$ Turner Institute for Brain and Mental Health, School of Psychological Sciences, Monash University, Clayton, Australia
${ }^{b}$ Monash Biomedical Imaging, Monash University, Clayton, Australia ${ }^{c}$ Orygen, Parkville, Australia
${ }^{d}$ Centre for Youth Mental Health, The University of Melbourne, Melbourne, Australia
${ }^{e}$ Melbourne Neuropsychiatry Centre, Department of Psychiatry, University of Melbourne
${ }^{f}$ Centre for Mental Health, Melbourne School of Global and Population Health, The University of Melbourne, Parkville, Australian
${ }^{g}$ School of Psychology, University of Birmingham, Edgbaston, UK Hospital Research Institute, University of Calgary, Calgary, Canada ${ }^{i}$ School of Physics, University of Sydney, New South Wales, Australia
${ }^{j}$ Centre for Complex Systems, University of Sydney, Camperdown, New South Wales, Australia \\ ${ }^{h}$ Departments of Radiology and Paediatrics, Hotchkiss Brain Institute and Alberta Children's
}

\begin{abstract}
Background Different regions of the brain's grey matter are connected by a complex structural network of white matter fibres, which are responsible for the propagation of action potentials and the transport of trophic and other molecules. In neurodegenerative disease, these connections constrain the way in which grey matter volume loss progresses. Here, we investigated whether connectome architecture also shapes the spatial pattern of longitudinal grey matter volume changes attributable to illness and antipsychotic medication in first episode psychosis (FEP).

Methods We conducted a triple-blind randomised placebo-control MRI study where 62 young adults with first episode psychosis received either an atypical antipsychotic or placebo over 6-months. A healthy control group was also recruited. Anatomical MRI scans were acquired at baseline, 3-months and 12-months. Deformation-based morphometry was used to estimate illness-related and antipsychotic-related grey matter volume changes over time. Representa-
\end{abstract}

\footnotetext{
* Corresponding Author

Email address: sid.chopra@monash.edu (Sidhant Chopra)
} 
tive functional and structural brain connectivity patterns were derived from an independent healthy control group using resting-state functional MRI and diffusion-weighted imaging. We used neighbourhood deformation models to predict the extent of brain change in a given area by the changes observed in areas to which it is either structurally connected or functionally coupled.

Results At baseline, we found that empirical illness-related regional volume differences were strongly correlated with predicted differences using a model constrained by structural connectivity weights $(\mathrm{r}=.541 ; \mathrm{p}<.001)$. At 3 -months and 12-months, we also found a strong correlation between longitudinal regional illness-related $(\mathrm{r}>.516 ; \mathrm{p}<.001)$ and antipsychotic-related volume change $(\mathrm{r}>$ $.591 ; \mathrm{p}<.001)$ with volumetric changes in structurally connected areas. These correlations were significantly greater than those observed across various null models accounting for lower-order spatial and network properties of the data. Associations between empirical and predicted volume change estimates were much lower for models that only considered binary structural connectivity (all $\mathrm{r}$ $<.376$ ), or which were constrained by inter-regional functional coupling (all $\mathrm{r}$ $<.436$ ). Finally, we found that potential epicentres of volume change emerged posteriorly early in the illness and shifted to the prefrontal cortex by later illness stages.

Conclusion Psychosis- and antipsychotic-related grey matter volume changes are strongly shaped by anatomical brain connectivity. This result is consistent with findings in other neurological disorders and implies that such connections may constrain pathological processes causing brain dysfunction in FEP.

\section{Introduction}

Psychotic disorders such as schizophrenia are characterised by anatomically widespread differences in grey matter volume $(\mathrm{GMV})^{1-7}$, with many of these differences showing evidence of progression as the illness evolves ${ }^{8-13}$. Metaand mega-analyses indicate that some of the most robust cross-sectional GMV differences are found in cingulate, frontal and temporal cortices, as well as medial temporal lobe, striatum and thalamus ${ }^{3-7,14-16}$, with longitudinal changes identified in frontal, temporal and parietal cortices $^{9,11}$. However, despite a large literature describing the location and nature of these brain changes, the specific mechanisms that give rise to their characteristic spatial pattern remain unknown.

The human brain is an intricate network of functionally specialised regions linked by a complex web of axonal fibres, referred to as a connectome ${ }^{17}$. These fibres enable the wide-spread coordination of neuronal dynamics and the transport of trophic and other biological molecules throughout the brain. They can also act as conduits for the spread of pathology, such that illness processes originating in one area can propagate to affect distributed systems via multiple mechanisms ${ }^{18,19}$. This principle has been powerfully demonstrated in dementia ${ }^{20-22}$, with evidence that GMV reductions in different neurodegenerative conditions appear to spread through the brain in a way that is constrained by the underlying architecture of the brain's white matter pathways. 
Recent work suggests that a network-based spreading process may also be involved in psychosis. Cross-sectional grey matter alterations correlate with increased fractional anisotropy in regionally adjacent white matter ${ }^{23}$ and grey matter differences in patients are often correlated across spatially distributed regions ${ }^{24-26}$. In recent work, Shafiei, et al. ${ }^{27}$ developed a simple neighbourhooddeformation model (NDM) in a sample of people with established schizophrenia that predicted the level of cross-sectional GMV reduction in an area based on the average reductions observed in other areas to which it was structurally connected. The analysis further revealed that transmodal frontal and cingulate cortices were putative epicentres of pathology, being regions associated with relatively large GMV reductions that were also connected to other areas showing large reductions.

Together, these findings support the hypothesis that the spatial patterning of GMV differences in psychotic illness is indeed constrained by connectome architecture. However, all studies addressing this question have been crosssectional, precluding an opportunity to track how coordinated grey matter changes evolve through time. Moreover, the reliance on samples of patients taking antipsychotic medication makes it difficult to determine whether coupled grey matter changes are driven by treatments for the illness or the illness processes. These effects can only be disentangled through a longitudinal, randomised placebo-controlled study of antipsychotic-naive patients. We recently used such a design to demonstrate that antipsychotic-medicated and non-medicated patients with First Episode Psychosis (FEP) show different trajectories of GMV change ${ }^{28}$ and functional coupling ${ }^{29}$ within the first 3 - and 12-months of treatment. Here, we use this cohort to investigate whether patterns of illness- and antipsychoticrelated GMV changes are constrained by the structural connectome and whether the strength of functional and/or structural coupling modulates this relationship. Using the $\mathrm{NDM}^{27}$, we show that coordinated patterns of GMV change in FEP are more tightly constrained by the extent of structural coupling (SC) than functional coupling (FC) between regions. Critically, the same pattern holds for both illness-related and antipsychotic-related longitudinal brain changes. We also track the dynamic progression of putative epicentres of illness-related GMV loss, showing that they emerge posteriorly early in the illness and shift to the prefrontal cortex by later illness stages. This pattern contrasts epicentres of antipsychotic-related GMV expansion, which are situated in primary visual and auditory cortices.

\section{Methods}

\section{Overview}

Our overall analysis strategy is outlined schematically in Fig. 1. Briefly, we used structural MRI images from a sample of FEP patients recruited to a placebo-controlled clinical trial of second-generation antipsychotics to estimate brain-wide, voxelwise illness-related and antipsychotic-related GMV changes at 
baseline, 3- and 12-months (Fig. 1A). We parcellated the brain into discrete regions-of-interest ${ }^{30,31}$ and estimated volume changes as the mean test-statistic of all voxels within that area. We used diffusion and functional MRI to generate group-level representative SC and FC matrices in an independent sample of healthy adults (Fig. 1B). These matrices were then used in three variants of the Neighbourhood Deformation Model (NDM) to evaluate the extent to which GMV differences in one area are coupled to the GMV differences observed in its structurally connected neighbours ${ }^{27}$. In the first variant, all neighbours make an equal contribution to predicting an index area's GMV. In the second and third variants, the contribution of each neighbour is scaled by either the weight of its structural or functional coupling to the index area, as determined by the representative SC and FC matrices, respectively. Model performance was evaluated using the Spearman correlation $(\rho)$ between region-wise estimates of observed and predicted volume changes (Fig. 1C). Finally, performance of each of the three NDM models was compared to three benchmark null models to ensure that our findings are not driven by lower order properties of the spatial maps or connectome architecture (Fig. 1D).
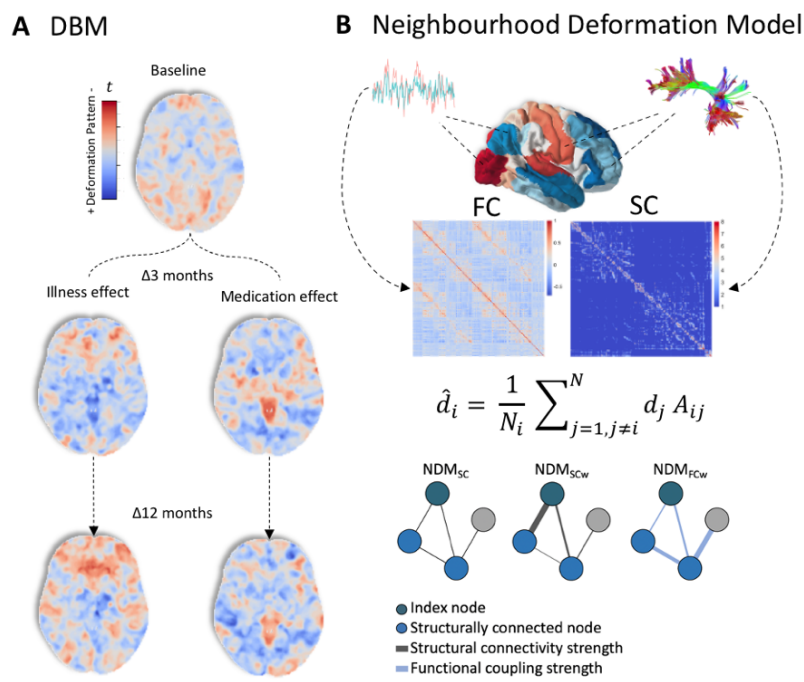

C Results

Fig 1. Analysis workflow for the Network Deformation Model. (A) We derived voxelwise GMV estimates using Deformation-based morphometry (DBM). Five separate contrasts were specified using a robust marginal model to infer baseline GMV differences and longitudinal GMV changes associated with illness and antipsychotic medication at 3-months and 12-months. (B) The contrast statistics were mapped to a brain parcellation comprising 332 regions, and diffusion and functional MRI data from an independent healthy sample were used to generate sample-averaged functional coupling (FC) and structural connectivity (SC) matrices. These matrices were used to model average volume changes in structurally connected neighbours. Under the NDM, the predicted 
deformation of a node, $\hat{d}_{i}$, is modelled as a weighted sum of the deformation values observed its structurally connected neighbours (shown as light blue nodes in the example graphs). The weights are given by the adjacency matrix, $A_{i j}$. Three different matrices were used, yielding three NDM variants; (1) A model denoted as $\mathrm{NDM}_{\mathrm{SC}}$, in which $A_{i j}=1$ if two regions share a connection and $A_{i j}=0$ otherwise; (2) a model denoted as $\mathrm{NDM}_{\mathrm{SCw}}$ in which the elements of $A_{i j}$ correspond precisely to the weighted SC matrix, such that the contribution of each neighbour is weighted by the strength of its structural connectivity to the index node; and (3) a model denoted $\mathrm{NDM}_{\mathrm{FCw}}$, in which the elements of $A_{i j}$ correspond precisely to the weighted FC matrix, such that the contribution of each neighbour is weighted by its level of FC with the index node. (C) Model performance was evaluated using the Spearman correlation between regional estimates of observed and predicted GMV differences. (D) We also compared model performance to three benchmark null models accounting for spatial autocorrelations in the deformation maps (Spatial ${ }_{\text {model }}$ and Spatial $\left.l_{\text {spin }}\right)$ and basic topological properties of the connectome (see Model evaluation).

\section{Sample characteristics}

\section{Clinical trial sample}

We recruited 62 people aged 15-25 years ( $46 \%$ female) who were experiencing FEP. All patients had minimal previous exposure to antipsychotic medication ( $<7$ days of use or lifetime $1750 \mathrm{mg}$ chlorpromazine equivalent exposure) and a duration of untreated psychosis of fewer than 6-months. At baseline, patients were randomised to one of two groups: one given antipsychotic medication (risperidone or paliperidone) plus intensive psychosocial therapy and the other given placebo plus intensive psychosocial therapy. For both groups, the treatment period spanned 6-months. MRI was conducted at baseline, 3-months, and 12-months post-intake. The randomisation phase of the study terminated at 6 months, so patients in either the antipsychotic or placebo group could have received antipsychotic medication and ongoing psychosocial interventions in between the 6- and 12-months into the study. A matched healthy control group comprising 27 individuals ( $63 \%$ female) with no history of psychiatric or neurological diagnosis was also recruited and scanned alongside the patient groups. Demographic details of this sample are provided in Table 1. Further sample characteristics and details about research and safety protocols can be found elsewhere ${ }^{32,33}$. Ethical approval for the study was granted by the Melbourne Health Human Research Ethics Committee (MHREC:2007.616).

\section{Independent healthy control sample}

A total of 119 healthy participants (66\% female) were recruited as part of a larger study conducted at Monash University. The participants were selected from a large cohort of 439 people as those with high-quality functional and diffusion MRI scans available and with an age that was within the range of the clinical trial sample. Participants were all right-handed, of European ancestry, and had no personal history of neurological or psychiatric disorders, had never 
suffered loss of consciousness or memory due to head injury, and did not have a history of drug abuse (for further details, see Sabaroedin, et al. ${ }^{34}$ ). The study was conducted in accordance with the Monash University Human Research Ethics Committee (MUHREC: 2012001562).

\begin{tabular}{|c|c|c|c|c|}
\hline & \multicolumn{2}{|c|}{ First episode psychosis } & \multirow[b]{2}{*}{$\begin{array}{l}\text { Matched healthy } \\
\text { control }(\mathrm{N}=27)\end{array}$} & \multirow[b]{2}{*}{$\begin{array}{l}\text { Independent } \\
\text { healthy control } \\
(\mathrm{N}=119)\end{array}$} \\
\hline & $\begin{array}{l}\text { Placebo } \\
(\mathrm{N}=30)\end{array}$ & $\begin{array}{l}\text { Antipsychotic } \\
(\mathrm{N}=29)\end{array}$ & & \\
\hline Baseline age, years (SD) & $18.8(2.72)$ & $19.5(2.94)$ & $21.9(1.93)$ & $20.9(1.68)$ \\
\hline Females, N (\%) & $14(46.6 \%)$ & $13(44.8 \%)$ & $17(62.9 \%)$ & $78(65.5 \%)$ \\
\hline \multicolumn{5}{|l|}{ Diagnosis, $\mathrm{N}$} \\
\hline Major depression with psychosis & 7 & 5 & & \\
\hline Schizophreniform disorder & 5 & 5 & & \\
\hline Psychotic disorder NOS & 8 & 7 & & \\
\hline $\begin{array}{l}\text { Substance-induced psychotic } \\
\text { disorder }\end{array}$ & 4 & 2 & & \\
\hline Delusional disorder & 1 & 4 & & \\
\hline Schizophrenia & 5 & 5 & & \\
\hline Missing diagnosis & 0 & 1 & & \\
\hline Baseline BPRS Total, mean (SD) & $59.4(9.64)$ & $55.8(10.10)$ & & \\
\hline Baseline SOFAS, mean (SD) & $52.9(14.0)$ & $51.7(10.6)$ & & \\
\hline
\end{tabular}

Table 1. Sample characteristics of clinical trial and healthy control sample. Abbreviations: NOS = not otherwise specified; BPRS = Brief Psychiatric Rating Scale version 4; SOFAS = Social and Occupational Functioning Assessment Scale.

\section{MRI acquisition}

\section{Clinical trial sample}

High resolution structural T1-weighted (T1w) scans were acquired using a 3-T Siemens Trio Tim scanner with a 32-channel head coil at the Royal Children's Hospital in Melbourne, Australia. Image acquisition parameters at each timepoint were as follow: 176 sagittal slices, with a $1 \mathrm{~mm}^{3}$ voxel size, bandwidth $236 \mathrm{~Hz} /$ pixel, field of view $(\mathrm{FOV})=256 \times 256 \mathrm{~mm}$, matrix $256 \times 256$, $2300 \mathrm{~ms}$ repetition time (TR), and 2.98 echo time (TE) and a $9^{\circ}$ flip angle. 


\section{Independent healthy control sample}

Structural, diffusion and functional MRI data were acquired using a Siemens Skyra 3T scanner with a 32-channel head coil at Monash Biomedical Imaging in Melbourne, Australia. T1w structural scans were acquired using: $1 \mathrm{~mm}^{3}$ isotropic voxels, $\mathrm{TR}=2300 \mathrm{~ms}, \mathrm{TE}=2.07 \mathrm{~ms}, \mathrm{TI}=900 \mathrm{~ms}$, and a FOV of $256 \mathrm{~mm}$.

Diffusion data were acquired using an interleaved acquisition with the following parameters: $2.5 \mathrm{~mm}^{3}$ voxel size, TR $=8800 \mathrm{~ms}, \mathrm{TE}=110 \mathrm{~ms}$, FOV $240 \mathrm{~mm}$, 60 directions with $\mathrm{b}=3000 \mathrm{~s} / \mathrm{mm}^{2}$, and seven $\mathrm{b}=0 \mathrm{~s} / \mathrm{mm}^{2}$ vol. In addition, a single $b=0 \mathrm{~s} / \mathrm{mm} 2$ was obtained with reversed phase encoding direction for susceptibility field estimation.

Multiband $\mathrm{T} 2 *$-weighted whole-brain echo-planar images were acquired with a total of 620 functional volumes with 42 slices each were acquired per participant using an interleaved acquisition with the following parameters: $\mathrm{TR}=754 \mathrm{~ms}$, $\mathrm{TE}=21$ milliseconds, flip angle of $50^{\circ}$, multiband acceleration factor of $3, \mathrm{FOV}$ $=190 \mathrm{~mm}$, slice thickness of $3 \mathrm{~mm}$, and $3 \mathrm{~mm}$ isotropic voxels. Participants were instructed to lie still in the scanner with eyes closed while maintaining wakefulness.

\section{Structural MRI processing}

Prior to processing, raw T1w scans were visually examined for artefacts and then subjected to an automated quality control procedure ${ }^{35}$. Three patient scans did not pass image quality control and were excluded due to image artefacts. The remaining scans were processed using the longitudinal deformationbased morphometry (DBM) pipeline of the Computational Anatomy Toolbox (version r1113) ${ }^{36}$ for the Statistical Parametric Mapping $12^{37}$ software running in Matlab version 2015a. For detailed information about DBM processing, see the Supplement. We used DBM to quantify volume changes because it requires less spatial smoothing ${ }^{38}$ than voxel-based morphometry (VBM) and to be comparable to previous work using the $\mathrm{NDM}^{27}$. We also replicated our primary findings using VBM, which has been used more extensively to quantify GMV in the literature (see Robustness analyses).

\section{Quantifying baseline and longitudinal grey matter changes in patients}

To estimate group-level baseline and longitudinal spatial patterns of volume change, we used a robust marginal model implemented in Sandwich Estimator Toolbox ${ }^{39}$, which combines ordinary least squares estimates of parameters of interest with estimates of variance/covariance based on a robust sandwich estimator, thus accounting for within-subject correlations across time. This method is asymptotically robust against misspecification of the covariance model and does not depend on the assumptions of common longitudinal variance structure across the whole brain. All contrasts were adjusted for age, sex and handedness.

We conducted three categories of contrasts (Fig. 1A): (1) illness-related differences at baseline, which compared all patients to healthy controls; (2) illnessrelated change over time, which compared GMV changes in the placebo group to 
healthy controls; and (3) antipsychotic-related changes, which compared GMV changes in the medication group to both the placebo group and healthy controls $\left(\right.$ see also $\left.{ }^{13}\right)$. Longitudinal contrasts were assessed from baseline to 3 -months, as well as from baseline to 12-months, with a linear polynomial contrast being used for the latter. The resulting t-statistics were converted to z-statistics. Contrasts were specified such that positive values in the resulting voxelwise z-statistic maps indicate lower volume in patients compared to controls at baseline contrast, and a greater longitudinal GMV decline in patients compared to controls in the longitudinal contrasts.

\section{Brain Parcellation}

In order to evaluate network-based models of grey matter changes, we parcellated the brain into 300 discrete cortical regions of approximately equal size ${ }^{31}$, in addition to 32 subcortical areas ${ }^{30}$, using previously published atlases. The deformation value for each region was estimated as the mean t-statistic of all voxels corresponding to that region. The regions comprise the nodes of a network, which can then be directly related to measures of inter-regional SC and FC.

\section{Healthy structural network}

We derived a group-level healthy structural connectome from diffusionweighted imaging (DWI) data from an independent sample of 119 adults (Fig. 1B). First, the DWI data for each individual were corrected using current best practise $^{40}$ for eddy-induced current distortions, susceptibility-induced distortions, intervolume head motion, outliers in the diffusion signal ${ }^{41}$, within-volume motion $^{42}$, and B1 field inhomogeneities. Probabilistic tractography was conducted using a standardised and reproducible pipeline ${ }^{43}$ and a state-of-the-art optimisation procedure was used to reduce false positive connections and improve biological interpretability of connection strength ${ }^{44}$. For a detailed overview of DWI processing and optimisation, see the Supplement. This procedure resulted in a single $332 \times 332$ weighted group-average SC matrix.

\section{Healthy functional network}

We derived a group-level healthy functional connectome from resting-state fMRI data from the same independent sample of adults (Fig. 1B). The fMRI data for each subject were processed using FSL FEAT ${ }^{45}$ and denoised using ICA-FIX $^{46,47}$. Detailed information on fMRI processing can be found in the Supplement. Given an ongoing controversy around the application of global signal regression ${ }^{48,49}$, we evaluated how this step affected our findings (see Robustness analyses). After processing and denoising, we computed a whole brain $332 \times 332$ FC matrix for each subject using pair-wise Pearson correlations between the timeseries from each of the 332 regions, and finally took a mean FC matrix across the sample. 


\section{Neighbourhood Deformation Model}

We evaluated network constraints on baseline and longitudinal GMV changes using the NDM introduced by Shafiei, et al. ${ }^{27}$. The model is given by

$$
\hat{d}_{i}=\frac{1}{N_{i}} \sum_{j=1, j \neq i}^{N_{i}} d_{j} A_{i j}
$$

where $\hat{d}_{i}$ is the predicted deformation in node $i, N_{i}$ is the number of structurally connected neighbours of $i, d_{j}$ is the deformation observed in the $j^{\text {th }}$ neighbour of node $i$, and $A_{i j}$ defines the connectivity between nodes $i$ and $j$.

Three different matrices were substituted for $A_{i j}$, yielding three variants of the NDM. For the first model, denoted $\mathrm{NDM}_{\mathrm{SC}}, A_{i j}=1$ if nodes $i$ and $j$ are connected in the group-average SC matrix and zero otherwise. As such, under this model, all $j$ structurally connected neighbours make an equal contribution to predicting the extent of deformation observed in node $i$.

For the second and third models, denoted $\mathrm{NDM}_{\mathrm{SCw}}$ and $\mathrm{NDM}_{\mathrm{FCw}}, A_{i j}$ corresponded to the weighted SC or FC matrices, respectively. Therefore, under these models, the contributions of node $i$ 's neighbours were weighted by either inter-regional $\mathrm{SC}\left(\mathrm{NDM}_{\mathrm{SC}}\right)$ or $\mathrm{FC}\left(\mathrm{NDM}_{\mathrm{FCw}}\right)$ estimates, such that neighbours of node $i$ with a more strongly weighted connection made a stronger contribution to predicting node $i$ 's volume (Fig 1B).

\section{Model evaluation}

Model performance was evaluated using the Spearman correlation $(\rho)$ between region-wise estimates of observed and predicted deformation (Fig. 1C). We also compared the performance of the $\mathrm{NDM}_{\mathrm{SC}}, \mathrm{NDM}_{\mathrm{SC}}$ and $\mathrm{NDM}_{\mathrm{FCw}}$ models to three benchmark null models.

The first (Fig. 1D; Spatial $\left.{ }_{\text {model }}\right)$ and second (Fig. 1D; Spatial $\left._{\text {spin }}\right)$ null models evaluated whether the observed findings were specific to the empirically observed pattern of grey matter deformations or were a generic property of the intrinsic spatial structure of the deformation maps. Specifically, we generated surrogate deformation maps with similar spatial structure to the empirical data in two ways. First, we used spatial variogram modelling to generate 1000 random spatial maps with a similar spatial autocorrelation to the observed deformation map, as implemented in the freely available toolbox BrainSMASH ${ }^{50}$; and with parameters $(n s=500 ; k n n=2300 ; p v=70)$ which resulted in nulls maps with variograms as close as possible to the empirical variogram. Second, we used a spin test to rotate region-level cortical t-values 1000 times $^{51}$. The rotation was applied to one hemisphere and then mirrored for the other hemisphere. The primary advantage of the model-based method is that it can be applied to both cortical and subcortical data, however, it is not guaranteed to match the precise spatial autocorrelation of the empirical data. The spin test exactly preserves the empirical values and their spatial autocorrelation but is only applicable to cortex. The 1000 surrogate values were used for inference on the observed performance 
metrics, with p-values quantified as the fraction of null values exceeding the observed correlation.

The third null model (Fig. 1D; Connectome) involved rewiring the structural connectome while preserving the degree sequence and length-weight relationship, and approximately preserving the edge-length distribution ${ }^{52}$. We used 10 distance bins and 50,000 edge swaps to generate 1000 rewired networks. These surrogate networks were used to test the hypothesis that any apparent network-based prediction of local grey matter change is specific to the actual topology of the connectome itself, and cannot be explained by basic network properties, such as regional variations in node degree or the spatial dependence of inter-regional connectivity.

Mapping epicentres of illness- and antipsychotic-related volume change

We next sought to identify putative epicentres of illness- and antipsychoticrelated volume changes. As per Shafiei, et al. ${ }^{27}$, we defined such epicentres as areas showing high deformation that were also connected to regions showing high deformation (Fig. 5A). To identify such regions, for each region and each contrast, we took the mean of two values: (1) that region's extent of deformation; and (2) the mean of that region's neighbours' deformation, weighted by SC as in the $\mathrm{NDM}_{\mathrm{SCw}}$ model, given the superior performance of this model (see Results). Higher positive values on the resulting epicentre score represent regions with high atrophy that are also connected to regions with high atrophy; lower negative epicentre scores represent regions with high expansion that are also connected to regions with high expansion (Fig. 5A). We then obtained a null ensemble of 1000 regional epicentre scores by repeating the same procedure after rotating the regional deformation maps relative to the structural connectome and retaining the absolute maximum null epicentre score detected across the brain. These 1000 null values were then used to quantify two-tailed family-wise error corrected statistical significance of each region's epicentre score as the fraction of null values exceeding the observed absolute epicentre score for a given regions ${ }^{53}$.

\section{Results}

Spatial distribution of illness- and antipsychotic-related volume deformations

We first mapped the spatial distribution of illness- and antipsychotic-related volume deformations using five separate contrasts (Fig. 1A). After mapping the voxelwise t-statistics to parcellated areas (Fig. 2A-C), we observed at baseline that patients showed relatively lower GMV primarily in the occipital cortex and left frontal pole, coupled with higher volume in the left middle frontal cortex (Fig. 2A). Illness-related decreases at 3-months (Fig. 2B) and 12-months (Fig. 2C) were largely consistent and most evident in frontal, temporal, cingulate, striatal and medial temporal regions, whereas increases were most evident within the thalamus, occipital and medial parietal cortex. Antipsychotic-related decreases 
at 3-months were most evident in superior parietal and temporal cortex, whereas increases were most evident in occipital cortex (Fig. 3A). At 12-months, antipsychotic-related decreases were most evident in superior parietal regions and increases were most evident in left cingulate and superior temporal cortex (Fig. 3B).

Structural connectivity shapes illness-related cross-sectional and longitudinal grey matter changes

We initially evaluated the performance of the three NDMs in capturing baseline differences in regional grey matter deformations. We observed the strongest correlation between predicted and observed estimates for the $\mathrm{NDM}_{\mathrm{SCw}}$ model $(\rho=.541$; Fig. 2G), which was nearly double the strength of the correlations observed for the $\mathrm{NDM}_{\mathrm{SC}}$ and $\mathrm{NDM}_{\mathrm{FCw}}$ models ( $\rho=0.320$ and $\rho=0.377$, respectively; Fig. 2D). The performance of the $\mathrm{NDM}_{\mathrm{SCw}}$ was also significantly better than all three benchmark models (all $\mathrm{p}<0.004$ ), whereas the $\mathrm{NDM}_{\mathrm{SC}}$ and $\mathrm{NDM}_{\mathrm{FC}_{\mathrm{w}}}$ models were only superior to the Spatial model $(\mathrm{p}<0.003)$, but not Spatial spin $_{\text {or }}$ connectome, benchmarks (all $\mathrm{p}>0.05$ ).

We observed the same pattern of findings for longitudinal illness-related changes at 3 -months and 12-months, with the $\mathrm{NDM}_{\mathrm{SC}}$ predictions correlating with empirical estimates at $\rho=0.516$ (Fig. 2H) and $\rho=0.550$ (Fig. 2I), respectively. The $\mathrm{NDM}_{\mathrm{SCw}}$ again showed significantly better performance than all three benchmark models (all $\mathrm{p}<0.034$ ). By comparison, correlations for the $\mathrm{NDM}_{\mathrm{SC}}$ and $\mathrm{NDM}_{\mathrm{FCw}}$ did not exceed $\rho=0.369$ and only showed significantly better performance than the Spatial $l_{\text {model }}\left(\right.$ Fig. 2E-F), but not Spatial ${ }_{\text {spin }}$ or connectome, benchmarks (all $\mathrm{p}>0.05$ ). 
A

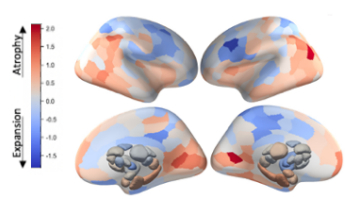

D

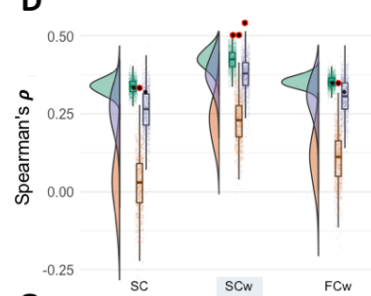

G

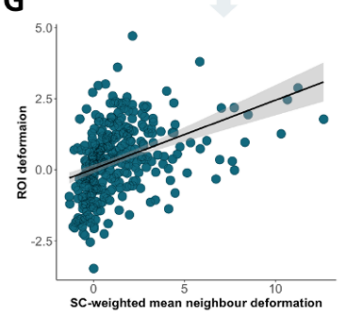

B

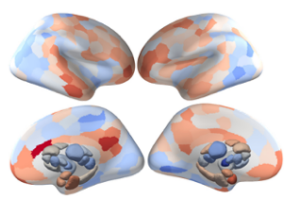

$\mathbf{E}$

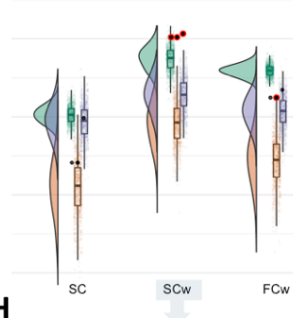

C $\Delta 12$ months

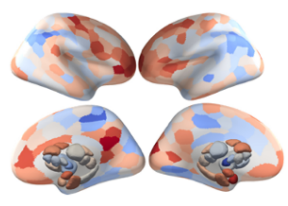

F

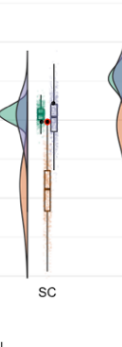

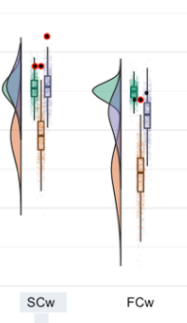

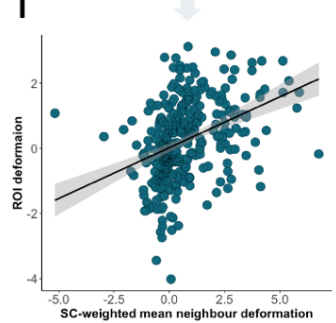

Null type

Connectome nulls Spatial model nulls Spatial spin nulls - Observed correlation $p<0.05$

Fig 2. Baseline and longitudinal illness-related GMV changes are constrained by connectome anatomy. A-C) The contrast statistics for three illness-related contrasts mapped to a brain parcellation comprising 332 regions. $\mathrm{D}-\mathrm{F}$ ) Performance of the $\mathrm{NDM}_{\mathrm{SC}}, \mathrm{NDM}_{\mathrm{SCw}}$ and $\mathrm{NDM}_{\mathrm{FCw}}$ models relative to the Spatial $_{\text {model }}$, Spatial $_{\text {spin }}$ and connectome null. Black circles indicate the observed correlations between predicted and actual regional deformation values for each model at each timepoint, with red borders indicating statistical significance $(\mathrm{p}<0.05)$. Note that the observed value used for comparison against the Spatial $_{\text {spin }}$ models is different because the subcortex was excluded. G-I) Scatterplots of the association between observed and predicted regional deformation values for the best performing $\mathrm{NDM}_{\mathrm{SC}}$ model at each timepoint.

Structural connectivity shapes longitudinal antipsychotic-related volume changes

As with illness-related changes, we found that the strongest correlation between observed and predicted antipsychotic-related grey matter changes was found using the $\mathrm{NDM}_{\mathrm{SCw}}$ model at both 3 -months $(\rho=0.591 ; \mathbf{F i g} .3 \mathbf{E})$ and 12 months $(\rho=0.604 ;$ Fig.3F). This association was statistically significant when compared to all three null models (all p $<0.007$; Fig.3C-D). For comparison, associations at 3 -months and 12 -months were smaller for the $\operatorname{NDM}_{\mathrm{SC}}(\rho=.376$ and $\rho=.174$, respectively) and $\mathrm{NDM}_{\mathrm{FCw}}$ models ( $\rho=.436$ and $\rho=.267$, respectively). At 3-months, the $\mathrm{NDM}_{\mathrm{SC}}$ and $\mathrm{NDM}_{\mathrm{FCw}}$ models only showed significantly better 
performance than the Spatial $l_{\text {model }}$ and Spatial $\mathrm{spin}_{\text {sin }}(\mathrm{p}<0.007)$ benchmarks. At 12-months, the $\mathrm{NDM}_{\mathrm{FCw}}$ model only showed significantly better performance than the Spatial model benchmark $(\mathrm{p}<0.014)$.

A

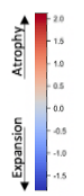

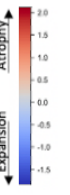

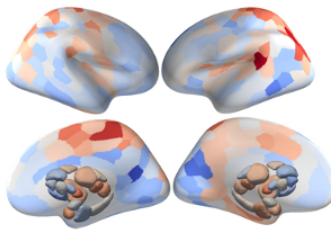

C

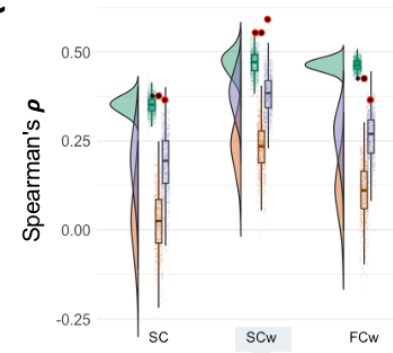

$\mathbf{E}$

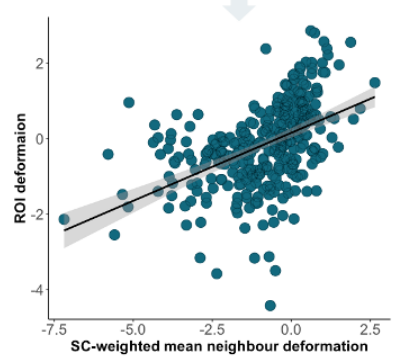

B $\Delta 12$ months

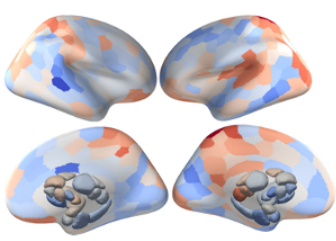

D

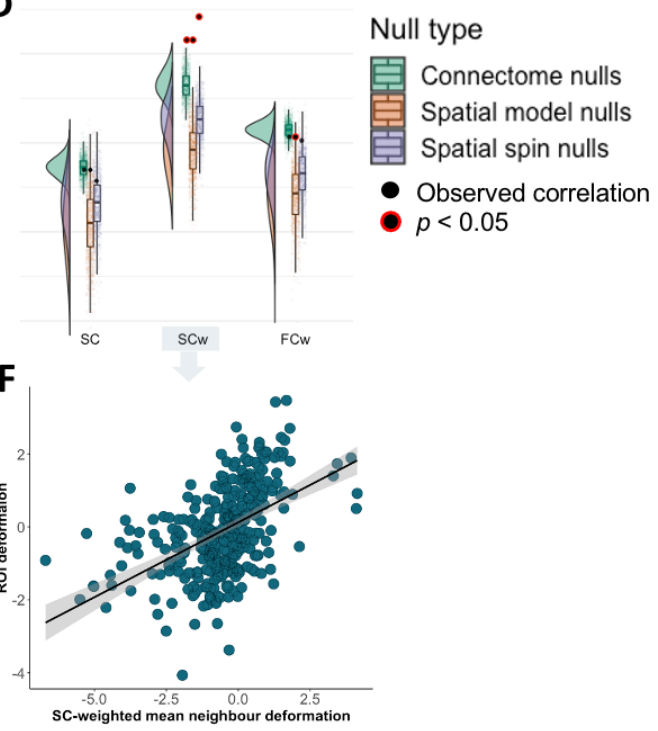

Fig 3. Longitudinal antipsychotic-related GMV changes are constrained by connectome anatomy. A-B) The contrast statistics for two antipsychotic-related contrasts mapped to a brain parcellation comprising 332 regions. C-D) Performance of the $\mathrm{NDM}_{\mathrm{SC}}, \mathrm{NDM}_{\mathrm{SC}}$ and $\mathrm{NDM}_{\mathrm{FC}}$ models relative to the Spatial $_{\text {model }}$, Spatial ${ }_{\text {spin }}$ and connectome null. Black circles indicate the observed correlations between predicted and actual regional deformation values for each model at each timepoint, with red borders indicating statistical significance $(\mathrm{p}<0.05)$. Note that the observed value used for comparison against the Spatial $_{\text {spin }}$ models is different because the subcortex was excluded. E-F) Scatterplots of the association between observed and predicted regional deformation values for the best performing $\mathrm{NDM}_{\mathrm{SC}}$ model at each timepoint. 


\section{Illness-related epicentres}

We next investigated regional epicentres of grey matter change, corresponding to areas that are connected to other areas showing a high degree of volume change patients in addition to showing large changes themselves. At baseline, 21 areas with statistically significant atrophy epicentre scores were identified in bilateral visual and left medial orbito-frontal cortex (Fig. 4D). At 3-months, areas with statistically significant atrophy epicentre scores were located within the left temporal pole and bilateral pre-frontal and orbitofrontal cortices (Fig. 4E). Areas with significant expansion epicentre scores were largely located in the visual cortex (Fig. 4E). At 12-months, the areas with significant regional epicentres extended across larger areas of anterior frontal cortex (Fig. $\mathbf{4 F}$ ).

A Epicentre Mapping

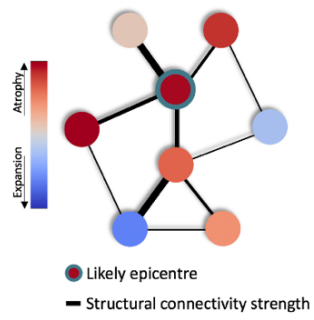

D

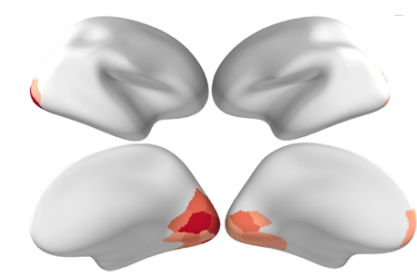

B Antipsychotic $-\Delta 3$ months

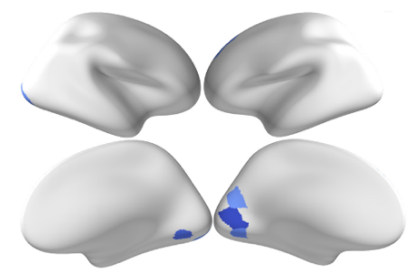

E Illness $-\Delta 3$ months

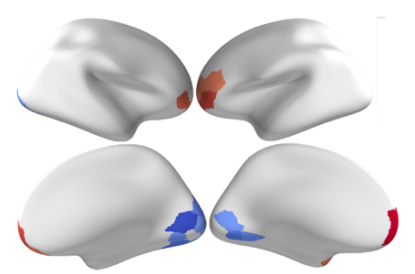

C Antipsychotic- $\Delta 12$ months

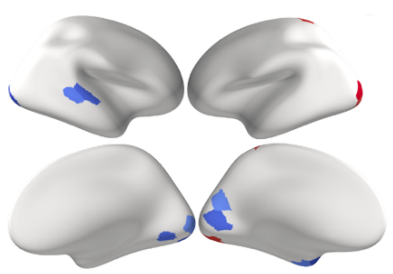

F IIIness $-\Delta 12$ months

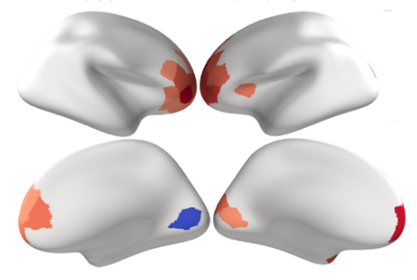

Fig 4. Regional epicentres of illness-related and medication-related GMV change. A) Epicentres were defined as areas showing high deformation that are also connected to regions showing high deformation. To identify such regions, for each region and each contrast, we computed a region-specific epicentre score as the mean of that area's deformation and the average of its neighbours' deformation, weighted by structural coupling strength. Regions showing epicentre scores significantly greater $\left(\mathrm{p}_{\mathrm{FWE}}<0.05\right)$ than a spatially constrained null model are shown for antipsychotic-related (A-B) and illness-related (D-F) effects.

\section{Antipsychotic-related epicentres}

At 3-months, we identified expansion-related epicentres in bilateral visual cortex (Fig 4B). At 12-months, additional expansion epicentres were located 
within the right superior temporal cortex and left temporal pole, along with a significant atrophy epicentre within the left occipital pole (Fig $\mathbf{4 C}$ ).

\section{Robustness analyses}

To ensure that our findings were not specific to the structural connectome derived from the large independent healthy control sample, we replicated our primary NDM analyses using a representative structural connectome derived from the matched healthy control sample which was part of the clinical trial. The magnitude and pattern of results remained consistent with our original illness and antipsychotic-related (Fig. S1) findings.

Next, we assessed whether our findings were specific to volume estimates derived using DBM by repeating our primary analyses using volume estimates from VBM. We found that the patterns of results for our primary analyses remained the same (Fig S2).

There can be several orders of magnitude difference between the deformation values and $\mathrm{SC} / \mathrm{FC}$ values. We therefore repeated the analyses after z-scoring the deformation values and connectivity weights and again found comparable results to our original findings (Fig. S3).

Finally, we assessed the impact of implementing global signal regression (GSR) on subject-level FC matrices before computing the group average FC matrix. We found that implementing GSR did not alter the patterns of results, with the $\mathrm{NDM}_{\mathrm{SCw}}$ model still showing the best performance (Fig. S4).

\section{Discussion}

Using a simple neighbourhood deformation model (NDM), we showed that the spatial pattern of both cross-sectional and longitudinal GMV changes in psychosis is shaped by brain network architecture, particularly by the strength of structural connectivity between regions. A similar relationship is apparent for GMV changes that are attributable either to psychotic illness or antipsychotic medication, indicating that the connectome represents a generic constraint on diverse processes driving coordinated grey matter variations. We also show a dynamic progression of regional epicentres of GMV change from posterior to anterior areas, suggesting that the pathological burden within prefrontal systems increases as the illness evolves.

\section{Network constraints on Illness-related volumes changes in FEP}

Our baseline findings in FEP align with the results of Shafiei, et al. ${ }^{27}$, who also used an NDM to show that structural connectivity constrains the spatial patterning of cross-sectional GMV differences in people with established schizophrenia. This earlier result was observed using the $\mathrm{NDM}_{\mathrm{SC}}$ model considered here. In our analysis, we found that the strength of structural connectivity between regions modulates coupled GMV differences within structurally connected neighbourhoods, given that the $\mathrm{NDM}_{\mathrm{SC}}$ showed clearly superior performance to 
the $\mathrm{NDM}_{\mathrm{SC}}$ and $\mathrm{NDM}_{\mathrm{FCw}}$ models. This result indicates that GMV differences are more tightly coupled between areas with high structural connectivity. This modulatory effect of the strength of structural connectivity may be especially pronounced early in the illness, as the performance of the binary $\mathrm{NDM}_{\mathrm{SC}}$ model in Shafiei, et al. ${ }^{27}$ analysis of patients with established schizophrenia was superior to $\mathrm{NDM}_{\mathrm{SC}}$ model performance in our sample. Critically, our findings show that network constraints on GMV differences cannot be explained by antipsychotic medication, as our FEP sample was antipsychotic-naïve at the baseline scan.

Moving beyond cross-sectional differences, our longitudinal analysis further demonstrates that both illness-related and antipsychotic-related changes in GMV are also constrained by connectome architecture. These results are in line with a spreading process, in which some pathological process propagates across axonal connections. The precise mechanisms driving this process remain unclear, but given the absence of evidence indicating an accumulation of pathological proteins in psychotic illness, it seems likely that the dysfunction of one region may trigger abnormal activity in connected sites which, over time, may trigger structural changes as a result of aberrant neurotransmission or a loss of trophic support ${ }^{19}$. This process may be exacerbated by a breakdown of white matter fibre integrity, which may further disrupt the inter-regional transport of trophic factors. Accordingly, widespread but subtle alterations in white matter have been repeatedly demonstrated in FEP populations ${ }^{55}$, are anticorrelated with cortical thickness ${ }^{23}$, and may predate the transition to psychosis in high-risk samples ${ }^{56,57}$. Further work may investigate how coordinated GMV changes relate to white matter pathology in patients. Given that we found that antipsychotic-related longitudinal changes in GMV are also constrained by connectome architecture, it is possible that neuronal signalling and/or neurotransmitter modulation induced by antipsychotics in one region may propagate to connected regions via structural connections.

Regional epicentres of grey matter change dynamically evolve with illness progression

Shafiei, et al.'s ${ }^{27}$ cross-sectional analysis of patients with established schizophrenia identified cingulate and prefrontal areas as putative epicentres of GMV reduction. Our analysis indicates that the involvement of prefrontal areas dynamically emerges as the illness progresses, shifting from an early posterior focus at the outset of illness. Progressively more prefrontal areas were identified as significant epicentres at 12-month compared to 3-month follow-up, suggesting that prefrontal circuits become increasingly compromised with ongoing illness. These findings align with longitudinal studies in early-onset schizophrenia demonstrating a dynamic wave of volume contraction progressing from posterior to anterior regions ${ }^{58,59}$, which may reflect an exaggeration of normal neurodevelopmental processes ${ }^{60}$. The findings also align with evidence for pronounced prefrontal GMV reductions in the earliest stages of illness ${ }^{8,61-66}$. Notably, these regional epicentres of illness-related GMV reduction localized to regions that were distinct from epicentres of antipsychotic-related GMV expansion, which were identified in primary visual and superior temporal cortex. The involvement of 
these regions may be related to antipsychotic-related improvements of perceptual abnormalities in patients ${ }^{67-69}$.

\section{Limitations and conclusions}

Given the complexity and practical challenges of conducting a prospective triple-blind randomised control MRI study in antipsychotic-naïve patients, the sample sizes within the patient groups are small. Relatedly, given the ethical and safety requirements of the study, patients who were recruited posed low risk of harm to self or others, lived in stable accommodation, had adequate social support and a short duration of untreated illness. While it's possible that these requirements may have resulted in a patient group with a less severe form of psychotic illness, the symptom severity and functioning of our patients (see Table 1) are comparable to epidemiologically representative cohorts of FEP patients $^{70,71}$.

The structural and functional connectomes used in this study were derived from healthy populations, in order to examine how normative network structure constrains the progression of GMV changes in FEP. Future studies should examine how altered structural connectivity in patients impacts our findings. Moreover, our findings are dependent on group-level summary metrics of brain volume and may not be representative of volume changes at the individual patient level, which show substantial heterogeneity ${ }^{72,73}$. Subsequent work could look at whether using individual-level measures of brain volume and connectivity can improve model predictions.

In summary, we show that longitudinal GMV changes in early psychosis are strongly constrained by the structural connectivity of the brain, but not its inter-regional functional coupling, regardless of whether those changes are driven by illness or antipsychotic medication. Our findings thus highlight a central role for axonal connectivity for shaping dynamic brain changes in the illness and suggest an increasing disruption of prefrontal systems with ongoing illness.

\section{Disclosures/Conflict of Interest}

$\mathrm{SF}, \mathrm{KA}, \mathrm{MAJ}$ and AF reported receiving grants from the Australian National Health \& Medical Research Council (NHMRC) during the conduct of the study. CP reported receiving grants from the Australian NHMRC and from the Lundbeck Foundation and personal fees from Lundbeck Australia Pty Ltd Advisory Board for talks presented at educational meetings organized by Lundbeck. PM reported receiving grants from the Australian NHMRC, the Colonial Foundation, the National Alliance for Research on Schizophrenia and Depression, the Stanley Foundation, the National Institutes of Health, Wellcome Trust, the Australian and Victorian governments, and Janssen-Cilag (unrestricted investigator-initiated grant) during the conduct of the study; past unrestricted grant funding from Janssen-Cilag, AstraZeneca, Eli Lilly, Novartis, and Pfizer; honoraria for consultancy and teaching from Janssen-Cilag, Eli Lilly, Pfizer, AstraZeneca, Roche, Bristol Myers Squibb, and Lundbeck. BN was supported by 
an NHMRC Senior Research Fellowship (1137687) and a University of Melbourne Dame Kate Campbell Fellowship. This work was supported by the computational infrastructure provided by the MASSIVE HPC facility (www.massive.org.au).

\section{References}

1 Gur, R. E., Turetsky, B. I., Bilker, W. B. \& Gur, R. C. Reduced Gray Matter Volume in Schizophrenia. Archives of General Psychiatry 56, 905-911, doi:10.1001/archpsyc.56.10.905 (1999). 2 Haijma, S. V. et al. Brain Volumes in Schizophrenia: A Meta-Analysis in Over 18000 Subjects. Schizophrenia Bulletin 39, 1129-1138, doi:10.1093/schbul/sbs118 (2013).

3 Steen, R. G., Mull, C., Mcclure, R., Hamer, R. M. \& Lieberman, J. A. Brain volume in first-episode schizophrenia. British Journal of Psychiatry 188, 510-518, doi:10.1192/bjp.188.6.510 (2006).

4 van Erp, T. G. M. et al. Cortical Brain Abnormalities in 4474 Individuals With Schizophrenia and 5098 Control Subjects via the Enhancing Neuro Imaging Genetics Through Meta Analysis (ENIGMA) Consortium. Biological Psychiatry 84, 644-654, doi:10.1016/j.biopsych.2018.04.023 (2018).

5 van Erp, T. G. M. et al. Subcortical brain volume abnormalities in 2028 individuals with schizophrenia and 2540 healthy controls via the ENIGMA consortium. Molecular Psychiatry 21, 547-553, doi:10.1038/mp.2015.63 (2016).

6 Gupta, C. N. et al. Patterns of Gray Matter Abnormalities in Schizophrenia Based on an International Mega-analysis. Schizophrenia Bulletin 41, 1133-1142, doi:10.1093/schbul/sbu177 (2015).

7 Vieira, S. et al. Neuroanatomical abnormalities in first-episode psychosis across independent samples: a multi-centre mega-analysis. Psychological Medicine 51, 340-350, doi:10.1017/s0033291719003568 (2021).

8 Pantelis, C. et al. Neuroanatomical abnormalities before and after onset of psychosis: a cross-sectional and longitudinal MRI comparison. The Lancet 361, 281-288, doi:10.1016/S0140-6736(03)12323-9 (2003).

9 Vita, A., De Peri, L., Deste, G. \& Sacchetti, E. Progressive loss of cortical gray matter in schizophrenia: a meta-analysis and meta-regression of longitudinal MRI studies. Translational Psychiatry 2, e190-e190, doi:10.1038/tp.2012.116 (2012).

10 Akudjedu, T. N. et al. Progression of neuroanatomical abnormalities after first-episode of psychosis: A 3-year longitudinal sMRI study. Journal of Psychiatric Research 130, 137-151, doi:10.1016/j.jpsychires.2020.07.034 (2020).

11 Olabi, B. et al. Are there progressive brain changes in schizophrenia? A metaanalysis of structural magnetic resonance imaging studies. Biological Psychiatry 70, 88-96, doi:10.1016/j.biopsych.2011.01.032 (2011).

12 Andreasen, N. C. et al. Progressive Brain Change in Schizophrenia: A Prospective Longitudinal Study of First-Episode Schizophrenia. Biological psychiatry 70, 672-679, doi:10.1016/j.biopsych.2011.05.017 (2011).

13 Chopra, S. et al. Differentiating the Effect of Medication and Illness on Brain 
Volume Reductions in First-Episode Psychosis: A Longitudinal, Randomized, Triple-blind, Placebo-controlled MRI study. medRxiv (2020).

14 Bora, E. et al. Neuroanatomical abnormalities in schizophrenia: A multimodal voxelwise meta-analysis and meta-regression analysis. Schizophrenia Research 127, 46-57, doi:10.1016/j.schres.2010.12.020 (2011).

15 Fornito, A., Yücel, M., Patti, J., Wood, S. J. \& Pantelis, C. Mapping grey matter reductions in schizophrenia: An anatomical likelihood estimation analysis of voxel-based morphometry studies. Schizophrenia Research 108, 104-113, doi:10.1016/j.schres.2008.12.011 (2009).

16 Del Re, E. C. et al. Anterior-posterior axis of hippocampal subfields across psychoses: A B-SNIP study. Biomarkers in Neuropsychiatry 5, 100037, doi:https: //doi.org/10.1016/j.bionps.2021.100037 (2021).

17 Sporns, O., Tononi, G. \& Kötter, R. The Human Connectome: A Structural Description of the Human Brain. PLOS Computational Biology 1, e42, doi:10.1371/journal.pcbi.0010042 (2005).

18 Raj, A. \& Powell, F. Models of Network Spread and Network Degeneration in Brain Disorders. Biological Psychiatry: Cognitive Neuroscience and Neuroimaging 3, 788-797, doi:10.1016/j.bpsc.2018.07.012 (2018).

19 Fornito, A., Zalesky, A. \& Breakspear, M. The connectomics of brain disorders. doi:10.1038/nrn3901 (2015).

20 Seeley, W. W., Crawford, R. K., Zhou, J., Miller, B. L. \& Greicius, M. D. Neurodegenerative diseases target large-scale human brain networks. Neuron 62, 42-52 (2009).

21 Zhou, J., Gennatas, E. D., Kramer, J. H., Miller, B. L. \& Seeley, W. W. Predicting regional neurodegeneration from the healthy brain functional connectome. Neuron 73, 1216-1227 (2012).

22 Raj, A., Kuceyeski, A. \& Weiner, M. A network diffusion model of disease progression in dementia. Neuron 73, 1204-1215, doi:10.1016/j.neuron.2011.12.040 (2012).

23 Di Biase, M. A. et al. Linking Cortical and Connectional Pathology in Schizophrenia. Schizophr Bull 45, 911-923, doi:10.1093/schbul/sby121 (2019).

24 Wannan, C. M. J. et al. Evidence for Network-Based Cortical Thickness Reductions in Schizophrenia. American Journal of Psychiatry, appi.ajp.2019.18040380, doi:10.1176/appi.ajp.2019.18040380 (2019).

25 Cauda, F. et al. The morphometric co-atrophy networking of schizophrenia, autistic and obsessive spectrum disorders. Hum Brain Mapp 39, 1898-1928, doi:10.1002/hbm.23952 (2018).

26 Jiang, Y. et al. Antipsychotics effects on network-level reconfiguration of cortical morphometry in first-episode schizophrenia. medRxiv, 2021.2001.2017.21249965, doi:10.1101/2021.01.17.21249965 (2021).

27 Shafiei, G. et al. Spatial patterning of tissue volume loss in schizophrenia reflects brain network architecture. Biological psychiatry 87, 727-735 (2020).

28 Chopra, S. et al. Differentiating the effect of antipsychotic medication and illness on brain volume reductions in first-episode psychosis: A Longitudinal, Randomised, Triple-blind, Placebo-controlled MRI Study. Neuropsychopharmacology 46, 1494-1501 (2021). 
29 Chopra, S. et al. Functional connectivity in antipsychotic-treated and antipsychoticnaive patients with first-episode psychosis and low risk of self-harm or aggression: a secondary analysis of a randomized clinical trial. JAMA psychiatry 78, 994$1004(2021)$.

30 Tian, Y., Margulies, D. S., Breakspear, M. \& Zalesky, A. Topographic organization of the human subcortex unveiled with functional connectivity gradients. Nature Neuroscience 23, 1421-1432, doi:10.1038/s41593-020-00711-6 (2020).

31 Schaefer, A. et al. Local-Global Parcellation of the Human Cerebral Cortex from Intrinsic Functional Connectivity MRI. Cerebral Cortex (New York, N.Y.: 1991) 28, 3095-3114, doi:10.1093/cercor/bhx179 (2018).

32 Francey, S. M. et al. Psychosocial Intervention With or Without Antipsychotic Medication for First-Episode Psychosis: A Randomized Noninferiority Clinical Trial. Schizophrenia Bulletin Open 1, doi:10.1093/schizbullopen/sgaa015 (2020). 33 O'Donoghue, B. et al. Staged treatment and acceptability guidelines in early psychosis study (STAGES): A randomized placebo controlled trial of intensive psychosocial treatment plus or minus antipsychotic medication for first-episode psychosis with low-risk of self-harm or aggression. Study protocol and baseline characteristics of participants. Early Intervention in Psychiatry 0, doi:10.1111/eip.12716 (2019).

34 Sabaroedin, K. et al. Functional Connectivity of Corticostriatal Circuitry and Psychosis-like Experiences in the General Community. Biological Psychiatry 86, 16-24, doi:10.1016/j.biopsych.2019.02.013 (2019).

35 Esteban, O. et al. MRIQC: Advancing the automatic prediction of image quality in MRI from unseen sites. PLOS ONE 12, e0184661, doi:10.1371/journal. pone.0184661 (2017).

36 Gaser, C. \& Dahnke, R. CAT - A Computational Anatomy Toolbox for the Analysis of Structural MRI Data. HBM, 336-348 (2016).

37 Ashburner, J. et al. SPM12 manual. Wellcome Trust Centre for Neuroimaging, London, UK 2464 (2014).

38 Schwarz, D. \& Kašpárek, T. Comparison of Two Methods for Automatic Brain Morphometry Analysis. Radioengineering 20 (2011).

39 Guillaume, B., Hua, X., Thompson, P. M., Waldorp, L. \& Nichols, T. E. Fast and accurate modelling of longitudinal and repeated measures neuroimaging data. NeuroImage 94, 287-302, doi:10.1016/j.neuroimage.2014.03.029 (2014).

40 Oldham, S. et al. The efficacy of different preprocessing steps in reducing motion-related confounds in diffusion MRI connectomics. NeuroImage 222, 117252, doi:https://doi.org/10.1016/j.neuroimage.2020.117252 (2020).

41 Andersson, J. L., Graham, M. S., Zsoldos, E. \& Sotiropoulos, S. N. Incorporating outlier detection and replacement into a non-parametric framework for movement and distortion correction of diffusion MR images. Neuroimage 141, 556-572 (2016).

42 Andersson, J. L. et al. Towards a comprehensive framework for movement and distortion correction of diffusion MR images: Within volume movement. Neuroimage 152, 450-466 (2017).

43 Al-Sharif, N. B., St-Onge, E., Theaud, G., Evans, A. C. \& Descoteaux, M. Processing the diffusion-weighted magnetic resonance imaging of the PING 
dataset. bioRxiv, 2020.2011.2024.396549, doi:10.1101/2020.11.24.396549 (2020). 44 Schiavi, S. et al. A new method for accurate in vivo mapping of human brain connections using microstructural and anatomical information. Science advances 6, eaba8245-eaba8245, doi:10.1126/sciadv.aba8245 (2020).

45 Woolrich, M. W., Ripley, B. D., Brady, M. \& Smith, S. M. Temporal autocorrelation in univariate linear modeling of FMRI data. Neuroimage 14, 1370-1386, doi:10.1006/nimg.2001.0931 (2001).

46 Salimi-Khorshidi, G. et al. Automatic denoising of functional MRI data: combining independent component analysis and hierarchical fusion of classifiers. Neuroimage 90, 449-468, doi:10.1016/j.neuroimage.2013.11.046 (2014).

47 Griffanti, L. et al. ICA-based artefact removal and accelerated fMRI acquisition for improved resting state network imaging. Neuroimage 95, 232-247, doi:10.1016/j.neuroimage.2014.03.034 (2014).

48 Murphy, K. \& Fox, M. D. Towards a consensus regarding global signal regression for resting state functional connectivity MRI. Neuroimage 154, 169-173, doi:10.1016/j.neuroimage.2016.11.052 (2017).

49 Aquino, K. M., Fulcher, B. D., Parkes, L., Sabaroedin, K. \& Fornito, A. Identifying and removing widespread signal deflections from fMRI data: Rethinking the global signal regression problem. Neuroimage 212, 116614 (2020).

50 Burt, J. B., Helmer, M., Shinn, M., Anticevic, A. \& Murray, J. D. Generative modeling of brain maps with spatial autocorrelation. NeuroImage 220, 117038 (2020).

51 Váša, F. et al. Adolescent tuning of association cortex in human structural brain networks. Cerebral Cortex 28, 281-294 (2018).

52 Betzel, R. F. \& Bassett, D. S. Specificity and robustness of long-distance connections in weighted, interareal connectomes. Proceedings of the National Academy of Sciences, 201720186, doi:10.1073/PNAS.1720186115 (2018).

53 Nichols, T. \& Hayasaka, S. Controlling the familywise error rate in functional neuroimaging: a comparative review. Statistical methods in medical research 12, 419-446 (2003).

54 Shafiei, G. et al. Global network structure and local transcriptomic vulnerability shape atrophy in sporadic and genetic behavioral variant frontotemporal dementia. bioRxiv (2021).

55 Yao, L. et al. White matter deficits in first episode schizophrenia: An activation likelihood estimation meta-analysis. Progress in Neuro-Psychopharmacology and Biological Psychiatry 45, 100-106, doi:https://doi.org/10.1016/j.pnpbp.2013. 04.019 (2013).

56 Di Biase, M. A. et al. White matter changes in psychosis risk relate to development and are not impacted by the transition to psychosis. Molecular Psychiatry, doi:10.1038/s41380-021-01128-8 (2021).

57 Carletti, F. et al. Alterations in White Matter Evident Before the Onset of Psychosis. Schizophrenia Bulletin 38, 1170-1179, doi:10.1093/schbul/sbs053 (2012).

58 Vidal, C. N. et al. Dynamically Spreading Frontal and Cingulate Deficits Mapped in Adolescents With Schizophrenia. Archives of General Psychiatry 63, 25-34, doi:10.1001/archpsyc.63.1.25 (2006). 
59 Thompson, P. M. et al. Mapping adolescent brain change reveals dynamic wave of accelerated gray matter loss in very early-onset schizophrenia. Proceedings of the National Academy of Sciences 98, 11650-11655, doi:10.1073/pnas.201243998 (2001).

60 Cropley, V. L. et al. Accelerated Gray and White Matter Deterioration With Age in Schizophrenia. Am J Psychiatry 174, 286-295, doi:10.1176/appi.ajp.2016. 16050610 (2017).

61 Sun, D. et al. Progressive Brain Structural Changes Mapped as Psychosis Develops in 'At Risk' Individuals. Schizophrenia research 108, 85-92, doi: 10.1016/j.schres.2008.11.026 (2009).

62 Sun, D. et al. Brain surface contraction mapped in first-episode schizophrenia: a longitudinal magnetic resonance imaging study. Molecular Psychiatry 14, 976-986, doi:10.1038/mp.2008.34 (2009).

63 Jalbrzikowski, M. Association of Structural Magnetic Resonance Imaging Measures With Psychosis Onset in Individuals at Clinical High Risk for Developing Psychosis: An ENIGMA Working Group Mega-analysis. JAMA Psychiatry 78, 753-766, doi:10.1001/jamapsychiatry.2021.0638 (2021).

64 Cannon, T. D. et al. Progressive reduction in cortical thickness as psychosis develops: a multisite longitudinal neuroimaging study of youth at elevated clinical risk. Biol Psychiatry 77, 147-157, doi:10.1016/j.biopsych.2014.05.023 (2015). 65 Chung, Y. et al. Cortical abnormalities in youth at clinical high-risk for psychosis: Findings from the NAPLS2 cohort. NeuroImage: Clinical 23, 101862, doi:https://doi.org/10.1016/j.nicl.2019.101862 (2019).

66 Del Re, E. C. et al. Baseline Cortical Thickness Reductions in Clinical High Risk for Psychosis: Brain Regions Associated with Conversion to Psychosis Versus Non-Conversion as Assessed at One-Year Follow-Up in the Shanghai-AtRisk-for-Psychosis (SHARP) Study. Schizophrenia Bulletin 47, 562-574 (2021). 67 Cadenhead, K., Dobkins, K., McGovern, J. \& Shafer, K. Schizophrenia spectrum participants have reduced visual contrast sensitivity to chromatic (red/green) and luminance (light/dark) stimuli: new insights into information processing, visual channel function, and antipsychotic effects. Frontiers in Psychology 4, doi:10.3389/fpsyg.2013.00535 (2013).

68 Grent-'t-Jong, T. et al. Association of Magnetoencephalographically Measured High-Frequency Oscillations in Visual Cortex With Circuit Dysfunctions in Local and Large-scale Networks During Emerging Psychosis. JAMA Psychiatry, doi:10.1001/jamapsychiatry.2020.0284 (2020).

69 Palaniyappan, L., Balain, V., Radua, J. \& Liddle, P. F. Structural correlates of auditory hallucinations in schizophrenia: A meta-analysis. Schizophrenia Research 137, 169-173, doi:https://doi.org/10.1016/j.schres.2012.01.038 (2012). 70 Leucht, S. et al. Clinical implications of Brief Psychiatric Rating Scale scores. British Journal of Psychiatry 187, 366-371, doi:10.1192/bjp.187.4.366 (2005).

71 Henry, L. P. et al. The EPPIC Follow-Up Study of First-Episode Psychosis: Longer-Term Clinical and Functional Outcome 7 Years After Index Admission. The Journal of Clinical Psychiatry 71, 716-728, doi:10.4088/JCP.08m04846yel (2010).

72 Wolfers, T. et al. Mapping the Heterogeneous Phenotype of Schizophre- 
nia and Bipolar Disorder Using Normative Models. JAMA Psychiatry, doi: 10.1001/jamapsychiatry.2018.2467 (2018).

$73 \mathrm{Lv}$, J. et al. Individual deviations from normative models of brain structure in a large cross-sectional schizophrenia cohort. Molecular Psychiatry 26, 3512-3523, doi:10.1038/s41380-020-00882-5 (2021).

74 Ashburner, J. A fast diffeomorphic image registration algorithm. NeuroImage 38, 95-113, doi:10.1016/j.neuroimage.2007.07.007 (2007).

75 Avants, B. B. et al. A reproducible evaluation of ANTs similarity metric performance in brain image registration. Neuroimage 54, 2033-2044, doi: 10.1016/j.neuroimage.2010.09.025 (2011).

76 Garyfallidis, E. et al. Dipy, a library for the analysis of diffusion MRI data. Frontiers in Neuroinformatics 8, doi:10.3389/fninf.2014.00008 (2014).

77 Tournier, J. D. et al. MRtrix3: A fast, flexible and open software framework for medical image processing and visualisation. NeuroImage 202, 116137, doi:https://doi.org/10.1016/j.neuroimage.2019.116137 (2019).

78 Basser, P. J., Mattiello, J. \& LeBihan, D. MR diffusion tensor spectroscopy and imaging. Biophys J 66, 259-267, doi:10.1016/s0006-3495(94)80775-1 (1994). 79 Tournier, J. D., Calamante, F. \& Connelly, A. Robust determination of the fibre orientation distribution in diffusion MRI: Non-negativity constrained super-resolved spherical deconvolution. NeuroImage 35, 1459-1472, doi:https: //doi.org/10.1016/j.neuroimage.2007.02.016 (2007).

80 Descoteaux, M., Deriche, R., Knösche, T. R. \& Anwander, A. Deterministic and probabilistic tractography based on complex fibre orientation distributions. IEEE Trans Med Imaging 28, 269-286, doi:10.1109/tmi.2008.2004424 (2009).

81 Ad-Dab'bagh, Y. et al. in Proceedings of the 12th annual meeting of the organization for human brain mapping. (Florence, Italy). 82 Theaud, G. et al. TractoFlow: A robust, efficient and reproducible diffusion MRI pipeline leveraging Nextflow \& Singularity. NeuroImage 218, 116889, doi:https: //doi.org/10.1016/j.neuroimage.2020.116889 (2020).

83 Girard, G., Whittingstall, K., Deriche, R. \& Descoteaux, M. Towards quantitative connectivity analysis: reducing tractography biases. Neuroimage 98 , 266-278, doi:10.1016/j.neuroimage.2014.04.074 (2014).

84 Smith, R. E., Tournier, J. D., Calamante, F. \& Connelly, A. Anatomicallyconstrained tractography: improved diffusion MRI streamlines tractography through effective use of anatomical information. Neuroimage 62, 1924-1938, doi:10.1016/j.neuroimage.2012.06.005 (2012).

85 Maier-Hein, K. H. et al. The challenge of mapping the human connectome based on diffusion tractography. Nature Communications 8, 1349, doi: 10.1038/s41467-017-01285-x (2017).

86 Schilling, K. G. et al. Limits to anatomical accuracy of diffusion tractography using modern approaches. Neuroimage 185, 1-11, doi:10.1016/j.neuroimage.2018. 10.029 (2019).

87 Betzel, R. F., Griffa, A., Hagmann, P. \& Mišić, B. Distance-dependent consensus thresholds for generating group-representative structural brain networks. Netw Neurosci 3, 475-496, doi:10.1162/netn_a_00075 (2019).

88 Jenkinson, M., Beckmann, C. F., Behrens, T. E., Woolrich, M. W. \& Smith, S. 
medRxiv preprint doi: https://doi.org/10.1101/2022.01.11.22268989; this version posted February 3, 2022. The copyright holder for this preprint (which was not certified by peer review) is the author/funder, who has granted medRxiv a license to display the preprint in perpetuity. It is made available under a CC-BY-NC-ND 4.0 International license.

M. FSL. Neuroimage 62, 782-790, doi:10.1016/j.neuroimage.2011.09.015 (2012). 